\title{
Kinematics, Free Energy and Dissipation in the Case of Transformation Induced Plasticity (TRIP)
}

\author{
M. Cherkaoui and M. Berveiller
}

Laboratoire de Physique et Mécanique des Matériaux, CNRS-ISGMP, Ile de Saulcy, 57045 Metz, France

\begin{abstract}
The aim of this work is to derive the behavior for an austenitic single crystal from which the overall behavior of polycrystalline TRIP steels can be deduced using classical scale transition method. The micromechanical modeling is based on the kinematics, kinetics and continuum-thermodynamics of the martensitic transformation. Plastic strains of product and parent phases as well as the volume fractions of each martensitic variants are taken as the internal variables describing the microstructure evolution of the single crystal. From the derived Helmboltz free energy and dissipation, we obtain the driving forces acting on these internal variables according to the irreversible thermodynamic formalism. First results are presented and discussed in the case of a thermomechanical shear test.
\end{abstract}

\section{INTRODUCTION}

The transformation induced plasticity deals with at least two inter related inelastic strain mechanisms: plastic flow by dislocation motion and the inelastic transformation strain related with the martensitic phase change. In addition to the usual thermoelastic properties which may be assumed homogeneous for usual TRIP materials like steels, the plastic behavior of an austenitic-martensitic two-phase material appears strongly heterogeneous. According to the diversity of active strain mechanisms and the evolutive microstructure, various couplings have to be described. From a kinematical point of view, a volume element suffers a plastic strain in its austenitic state, followed by the instantaneous transformation strain according to discrete values of the possible set of transformation strains and plastic flow in the martensitic state. Beside the chemical part of the free energy, the elastic part is due to applied stresses but also to internal stresses generated by the incompatibilities of the whole inelastic strain field (plastic+transformation). These couplings lead to two well known effects [1]:

- the Magee [2] effect (or orientation effect) corresponds to the orientation of the transformation (or to the variant selection) by internal as well as by applied stresses,

- The Greenwood-Johnson [3] effect (or accommodation effect) is related with accommodation by oriented plastic flow of austenitic and martensitic phases due to the local stress field inside both phases.

Except some phenomenological laws and finite element calculations $[4,5,6,7,8]$, the study of the constitutive equation for the TRIP steels is yet much developed as compared with shape memory alloys.

The aim of this work is to derive constitutive equations for an austenitic single crystal undergoing martensitic phase transformation within a thermodynamic and micromechanic framework taking into account both the orientation effect (Magee effect) and the accommodation effect (Greenwood-Johnson effect). The progress of the transformation is assumed to result from the nucleation and instantaneous growth of new martensitic domains considered as ellipsoidal inclusions. The Helmholtz free energy and its evolution including the chemical part are deduced from a micromechanical analysis in which the elastic part is obtained using simultaneously the classical plastic inclusion problem together with interface operators. The theoretical model is applied in a simple case corresponding to a macroscopic shear where only one martensitic variant is assumed to appear together with simple slip in austenite as well as in martensite.

\section{KINEMATICS AND PHASE TRANSFORMATION KINETICS}

The continuum mechanic aspects of a thermomicromechanical approach for the behavior of heterogeneous materials are usually based on the description of a representative volume element (RVE), which contains enough microscale deformation information. Let us consider the austenitic single crystal as a RVE with volume $V$ and external surface $\partial V$. The reference configuration consists of a pure austenitic phase assumed to be stress free. The current configuration corresponds to the volume $V$ under a thermomechanical load, a temperature $T$ and uniform stress $\Sigma$ (or uniform strain $E$ ). At this stage of transformation a certain number $\mathrm{N}$ of 24 crystallographically possible variant $I$ with corresponding stress free transformation strains $\varepsilon^{\text {tr } I}$ has 
developed with volumes $V I$, boundaries $S I$ and corresponding volume fractions $f I=V I / V$. The typical shape of a martensitic domain (or variant) is a plate or lath with small ratio of thickness to other dimensions. The martensitic transformation consists of nucleation and growth processes corresponding to boundary motions of martensitic domains $V I$ whose velocities are different from those of material particles. Consequently, the kinetics of the martensitic transformation can be described by the volume fraction rate which is linked to the velocity $w^{I}$ of the boundary $S^{I}$ through the following relation

$$
\dot{f}^{I}=\frac{1}{V} \int_{S I} w_{\alpha}^{I} n_{\alpha}^{I} d S^{I},
$$

where $n_{\alpha}^{I}$ are the components of the unit normal vector to the boundary $S I$. Each variant is characterized by an habit plane normal $\boldsymbol{N}$, a direction of transformation $\boldsymbol{M}$ and the amplitude $g$ of the transformation strain considered as a material constant. Starting from the crystallographic theory of the martensitic transformation [9], we can calculate the transformation strain $\varepsilon^{\mathbb{t} I}$ of the variant $I$ as

$$
\varepsilon_{i j}^{\mathrm{tr} I}=g R_{i j}^{I}=\frac{1}{2} g\left(M_{i}^{I} N_{j}^{I}+M_{j}^{I} N_{i}^{I}\right),
$$

where $R^{I}$ is called the orientation tensor of the martensitic variant $I$.

In case of TRIP steels, the deformation field results from several strain mechanisms:

- an elastic part $\varepsilon^{\mathrm{e}}(r)$ due to the elastic deformation of martensitic and austenitic lattices,

- a plastic part $\varepsilon \mathrm{p}(r)$ due to the plastic flow of parent and product phases associated with creation, annihilation and movement of dislocations (slip),

- the transformation strain $\varepsilon^{\text {tr }}(r)$ due to the inelastic change of lattices during phase transformation and lattice invariant strain,

- the thermal strain $\mathcal{E}^{\text {th }}(r)$ which is usually low compared with the other strains and therefore neglected in the following. With small strain hypothesis, the total strain can be decomposed as

$$
\varepsilon(r)=\varepsilon^{e}(r)+\varepsilon^{\mathrm{p}}(r)+\varepsilon^{\mathrm{tr}}(r) .
$$

The total macroscopic strain $E$ of the RVE is the average over the volume $V$ of the local strain $\varepsilon(r)$.

$$
E=\frac{1}{V} \int_{V} \varepsilon(r) d V .
$$

If we assume that the martensite and austenite phases have the same elastic tensor $C$, equation (4) leads to

$$
E=E \mathrm{e}+E \mathrm{p}+E^{\mathrm{t}},
$$

where $E \mathrm{p}$ and $E^{\mathrm{tr}}$ are respectively the macroscopic plastic and transformation parts of the overall strain defined by

$$
E \mathrm{p}=\frac{1}{V} \int_{V} \varepsilon \mathrm{p}(r) d V=(1-f) \bar{\varepsilon} \mathrm{p}_{\mathrm{A}}+f \bar{\varepsilon} \mathrm{pM}, \quad E \mathrm{tr}=\frac{1}{V} \int_{V} \varepsilon^{\mathrm{tr}}(r) d V=\sum_{I} f^{I} \mathcal{E}^{\mathrm{tr} I}=g \sum_{I} f^{I} R^{I}
$$

and $E \mathrm{e}$ the elastic part linked to the overall stress $\Sigma$ using the linear elasticity $(E \mathrm{e}=C-1 \Sigma) . \bar{\varepsilon} \mathrm{p}_{\mathrm{A}}$ is the average plastic strain over the austenitic phase, $\bar{\varepsilon}$ pM is the one over the martensitic phase and $f$ its total volume fraction. It will be necessary to follow not only the mean value of plastic strain inside the volume $V M$ of martensite but also for each martensitic variant (volume $V^{I}$ ) so that we can write

$$
f \bar{\varepsilon} \mathrm{pM}=\sum_{I} f I \overline{\mathcal{E}}^{\mathrm{M} I},
$$

where $\bar{\varepsilon} M_{I}$ is the mean plastic strain over the volume of the variant $I$ composed by a set of elementary martensitic domains. In fact, the plastic strains of each martensitic domain belonging to one variant are different since the domains are formed at various times where the plastic strain inside the austenite is 
different. From the current stage, a thermomechanical loading (an imposed stress rate $\Sigma$ and a uniform temperature $\dot{T}$ ) lead to the formation of new martensitic domains with an instantaneous growth as well as to the plastic flow of the austenitic phase and the pre-existing martensitic domains. The elastic overall response $\dot{E}^{\mathrm{e}}$ of the RVE is known. In the following our attention is focused on the evolution of the inelastic part $E \mathrm{tp}=E \mathrm{p}+E$ tr which is the mean value of the inelastic local strain $\varepsilon^{\mathrm{tp}}(r)=\varepsilon \mathrm{p}(r)+\varepsilon^{\mathrm{tr}}(r)$

$$
E \mathrm{tp}=\frac{1}{V} \int_{V} \varepsilon^{\mathrm{tp}}(r) d V
$$

The inelastic response $\dot{E}$ tp due to the thermomechanical loading $\dot{\Sigma}$ and $\dot{T}$ can be calculated through the time derivative of (8) by taking into account the jump of the local inelastic strain $\varepsilon t(r)$ across the boundaries $S I$. We obtain the following form

$$
\dot{E} \mathrm{tp}=\frac{d}{d t}\left(\frac{1}{V} \int_{V} \varepsilon^{\mathrm{tp}}(r) d V\right)=\frac{1}{V} \int_{V} \dot{\varepsilon} \mathrm{tp}(r) d V-\frac{1}{V} \sum_{I} \int_{S^{I}}\left[\varepsilon^{\mathrm{tp}}(r)\right]^{I} w_{\alpha}^{I} n_{\alpha}^{I} d S^{I},
$$

where $\dot{\varepsilon}^{\mathrm{tp}}(r)=\partial \varepsilon^{\mathrm{tp}}(r) / \partial t$ is the rate of the local inelastic strain and $\left[\varepsilon^{\mathrm{tp}}(r)\right]^{I}$ its jump across the moving boundaries $S I$ corresponding to the formation of new martensitic domains. With the instantaneous growth assumption and after some average operations, equation (9) is reduced to

$$
\dot{E}_{i j}^{\mathrm{tp}}=(1-f) \sum_{n} P_{i j}^{\mathrm{A}_{n}} \dot{\gamma}^{n}+\sum_{I, n} f^{I} P_{i j}^{I n} \dot{\gamma}^{I n}+\sum_{I} \varepsilon_{i j}^{\mathrm{t} I} \dot{f}^{I}
$$

where $\dot{\gamma}^{n}$ and $\dot{\gamma}^{I n}$ are the plastic slip rates on glide systems $n$ of austenite and martensitic variants, respectively. $P A_{n}$ and $P I_{n}$ are the corresponding Schmid tensors.

Equation (10) shows the nature of internal variables describing the evolution of the RVE microstructure associated with the inelastic behavior: the volume fraction of each possible martensitic variant, the plastic strain of the austenitic phase and the plastic strain of the martensitic phase.

The evolution of these internal variables as function of the thermomechanical load are derived using free energy and dissipative concepts taking into account the dissipation due to the plastic flow as well as to the frictional work due to boundary motions. These allows to obtain the thermodynamic driving forces acting on internal variables and therefore the conditions for the formation of martensitic variants as well as for the plastic flow of product and parent phases. Furthermore, an additional internal variable appears in thermodynamic driving forces (see section 3.) and therefore plays a key role in plasticity and phase transformation conditions. This internal variable is the average plastic strain $\bar{\varepsilon}^{M_{I}}$ over a martensitic variant $I$ whose evolution is given by

$$
f I \dot{\bar{\varepsilon}}_{i j}^{\mathrm{M} I}=f^{I} \sum_{n} P_{i j}^{I_{n}} \dot{\gamma} I n+\dot{f}^{I}\left(\bar{\varepsilon}_{i j}^{\mathrm{pA}}-\bar{\varepsilon}_{i j}^{\mathrm{M} I}\right) .
$$

\section{HELMHOLTZ FREE ENERGY AND THERMODYNAMIC DRIVING FORCES}

At the current stage Helmholtz free energy $\Phi$ per unit volume of the martensitic-austenitic system includes three different contributions : the chemical free energy, surface energy and elastic strain energy. The surface energy due to the presence of martensitic-austenitic interfaces related with the formation of martensitic variants is generally weak in first order martensitic transformations, and therefore, disregarded in this work. Furthermore, the coupling between stress and temperature is not considered in this work, because the change in mechanical properties of two phases with temperature is low compared to the change in chemical energy with respect to temperature. From this point of view, $\Phi$ can be written as a sum of a term directly coupled with temperature and term directly coupled with stress :

- The temperature dependent term describes the chemical free energy $\Phi$ ch per unit volume of the martensitic-austenitic system given by

$$
\Phi \mathrm{ch}=\frac{1}{V} \int_{V} \varphi(r) d V
$$


- The stress dependent term corresponds to the elastic strain energy

$$
W=\frac{1}{V} \int_{V} w(r) d V
$$

where $\varphi(r)$ and $w(r)$ are the chemical and elastic strain energy densities, respectively. From (12) and (13) the Helmholtz free energy per unit volume of the austenitic-martensitic system is given by

$$
\Phi=W+\Phi \mathrm{ch}=\frac{1}{V} \int_{V}(w(r)+\varphi(r)) d V .
$$

At the current stage, the evolution of the Helmholtz free energy associated with the change of the thermomechanical loading results from the progression of the plastic strain of parent and product phases as well as from the moving boundaries related with the formation of new martensitic domains. As described previously, the stress and strain field are discontinuous across the moving boundaries and therefore the elastic strain energy density too. Due to the difference of the crystal structure between austenite and martensite, the chemical energy density is also discontinuous. Thus, the jumps $[w(r)]$ and $[\varphi(r)]$ of elastic and chemical energies have to be taken into account for the calculation of the Helmholtz free energy evolution. By the time derivative of (14), it follows that

$$
\dot{\Phi}=\frac{1}{V} \int_{V}(\dot{w}(r)+\dot{\varphi}(r)) d V-\frac{1}{V} \sum_{I} \int_{S^{I}}\left([w(r)]^{I}+[\varphi(r)]^{I}\right) w_{\alpha} n_{\alpha} d S^{I}
$$

The volumic variation in (15) is due to the plastic strain mechanism related with the plastic flow of austenitic phase and the pre-existing martensitic variants. The surface integral corresponds to the transformation strain mechanisms associated with the formation of new martensitic domains. With uniform elasticity and using the behavior laws in both side of the austenitic-martensitic boundary, (15) gives

$$
\begin{aligned}
& \dot{\Phi}=\frac{1}{V} \int_{V} \sigma_{i j}(r)\left(\dot{\varepsilon}_{i j}(r)-\dot{\varepsilon}_{i j}^{\mathrm{p}}(r)\right) d V- \\
& \frac{1}{V} \sum_{I} \int_{S I}\left(\frac{1}{2}\left\{\sigma_{i j}^{\mathrm{A} I}\left(r^{+}\right)+\sigma_{i j}^{\mathrm{M} I}\left(r^{-}\right)\right\}\left[\varepsilon_{i j}(r)-\varepsilon_{i j}^{\mathrm{p}}(r)\right]^{I}-B\left(T-T^{\circ}\right)\right) w_{\alpha} n_{\alpha} d S_{I},
\end{aligned}
$$

where $B$ and $T^{\circ}$ are a material constants. $\sigma^{A} I\left(r^{+}\right)$and $\sigma^{M}{ }_{I}\left(r^{-}\right)$are the limit values of the local stress on $S I$ from the austenitic and martensitic sides, respectively.

On the other hand, the power $P$ e developed by external forces $T_{i}=\Sigma_{i j} n_{j}$ applied on the external boundary $\partial V$ of the RVE in the absence of volumic forces is defined by

$$
P^{e}=\frac{1}{V} \int_{\partial V} T_{i} v_{i} d S=\frac{1}{V} \int_{\partial V} \Sigma_{i j} n_{j} v_{i} d S,
$$

where $v_{i}=\dot{u}_{i}$ are the components of the particle velocity vector and $n_{i}$ components of the unit normal vector to the boundary $\partial \mathrm{V}$. Using the divergence theorem with the Hadamard relations and taking into account the equilibrium equations in absence of volumic forces, the power $P$ e is expressed as

$$
P \mathrm{e}=\frac{1}{V} \int_{V} \sigma_{i j}(r) \dot{\varepsilon}_{i j}(r) d V-\frac{1}{2 V} \sum_{I} \int_{S I}\left(\sigma_{i j}^{\mathrm{A} I}\left(r^{+}\right)+\sigma_{i j}^{\mathrm{M} I}(r-)\right)\left[\varepsilon_{i j}(r)\right]^{I} w_{\alpha} n_{\alpha} d S I
$$

During the evolution of the austenitic-martensitic system, the intrinsic dissipation is given by the difference between the power developed by the external forces and the variation of the Helmholtz free energy. From (16) and (17) and using the instantaneous growth hypothesis, one finds the dissipation $D$ as

$$
D=\frac{1}{V} \int_{V} \sigma_{i j}(r) \dot{\varepsilon}_{i j}^{\mathrm{p}}(r) d V+\frac{1}{V} \sum_{I} \int_{S I}\left(\frac{1}{2}\left\{\sigma_{i j}^{\mathrm{A} I}\left(r^{+}\right)+\sigma_{i j}^{\mathrm{M} I}\left(r^{-}\right)\right\} \varepsilon_{i j}^{\mathrm{tI} I}-B(T-T \circ)\right) w_{\alpha} n_{\alpha} d S^{I} .
$$


The first term in (19) is the volume dissipation due to the evolution of austenitic and martensitic plastic strains. The second term corresponds to the propagation of boundaries $S I$ related with the formation of martensitic domains. Furthermore, these terms show the local thermodynamical driving forces associated with the microscale strain mechanisms describing the evolution of the RVE microstructure :

- the local stress $\sigma(r)$ as the driving force for the plastic strain mechanism. This stress field results from the applied stress as well as from the internal stress generated both by the plastic and transformation strains, - the term $\left(1 / 2\left\{\sigma \mathrm{A}_{I}\left(r^{+}\right)+\sigma \mathrm{MI}_{I}(r-)\right\} \varepsilon^{\mathrm{tr} I}-B(T-T \mathrm{o})\right)$ as the local driving force for the transformation strain mechanism which can be also defined as the driving force acting on the boundary $S^{I}$ of a martensitic domain during its instantaneous growth. The determination of driving forces associated with plastic and transformation strain mechanisms requires the knowledge of the stress distribution in austenitic phase and martensitic variants. This is accomplished by using simultaneously the concepts of ellipsoidal inclusion, interfacial operators [10] and homothetic ellipsoidal growing [11]. If we denote by $\sigma$ A the average stress in austenitic phase and $\sigma \mathrm{M}_{I}$ the one in a martensitic variant $I$, one obtains:

- the driving forces as the resolved shear stress on slip systems in austenite and martensitic variants $I$

$$
\begin{gathered}
\tau \mathrm{A}=\sigma \mathrm{A}^{\mathrm{A} \mathrm{A}_{n}}=\left(\Sigma-\sum_{I} f^{I} C(I-S I)\left(\bar{\varepsilon} \mathrm{p}_{\mathrm{A}}-\varepsilon^{\mathrm{tr} I}-\bar{\varepsilon} \mathrm{M}_{I}\right)\right) P_{\mathrm{A}_{n}} \\
\tau \mathrm{M}_{I}=\sigma \mathrm{M}_{I} P I_{n}=\left(\Sigma+C\left(I-S^{I}\right)\left(\bar{\varepsilon} \mathrm{p}_{\mathrm{A}}-\varepsilon^{\mathrm{tr}_{I}}-\bar{\varepsilon}^{\mathrm{M}_{I}}\right)-\sum_{J} f^{J} C\left(I-S^{J}\right)\left(\bar{\varepsilon} \mathrm{pA}_{\mathrm{A}}-\varepsilon^{\mathrm{tr} J}-\bar{\varepsilon}^{\mathrm{M} J}\right)\right) P I_{n},
\end{gathered}
$$

- the driving force for the formation of a volume fraction $\dot{f}^{I}$

$$
\tau^{f_{I}}=\left(\sigma^{\mathrm{A}}-\frac{1}{2} C\left(I-S^{I}\right) \varepsilon^{\mathrm{t}_{I}}\right) \varepsilon^{\mathrm{t}_{I}}-B\left(T-T^{\mathrm{o}}\right)
$$

with $\varepsilon^{\text {tr } I}$ as transformation strain and martensitic domains with Eshelby tensor $S I$. Equation (22) contains the extended Magee effect since $\sigma \mathrm{A}$ depends on applied as well as internal stresses. Simultaneously, equations (20) and (21) represents the Greenwood-Johnson effect since $\sigma \mathrm{A}$ and $\sigma \mathrm{M}_{I}$ depends on the internal stresses generated by the martensitic phase transformation.

\section{CRITICAL FORCES AND OVERALL BEHAVIOR}

For thermodynamic irreversible processes corresponding to plastic flow and martensitic transformation the obtained driving forces $\tau_{i}\left(\Sigma, T ; X_{j}\right)$ have to be compared with critical forces $\tau_{i}^{c}\left(X_{i}\right)$ depending on internal variables $X_{i}$. The expression of these critical forces is based on physical concepts taking into account :

- the hardening of austenite and martensite due to their own plastic strains,

- the hardening of the martensitic phase due to the plastic strain inherited from the austenite,

- the fact that the plastic flow of the austenitic phase assists the martensitic phase transformation (strain induced martensitic transformation),

- the martensitic topological hardening related with geometrical restrictions traducing the fact that the growth of martensitic domains is limited by grain boundaries as well as by already existing martensitic variants.

We assume that the inelastic mechanisms related with plasticity and phase transformation are of time independent type so that any viscous effect is neglected. In that case, the evolution of critical forces as function of internal variables can be expressed such as

$$
\tau_{i}^{c}\left(X_{k}\right)=H i j \dot{X}_{j}
$$

where $H i j=\partial \tau_{i}^{c}\left(X_{k}\right) / \partial X_{j}$ are the interaction matrices between the different internal variables $X_{i}$ which can be determined using the physical concepts discussed previously. The consistency rule allows to obtain the following non-linear system for the case where the equalities $\tau_{i}\left(\Sigma, T, X_{j}\right)=\tau_{i}^{\mathrm{c}}\left(X_{j}\right)$ are satisfied

$$
\left(\frac{\partial \tau_{i}^{c}}{\partial X_{j}}\left(X_{j}\right)-\frac{\partial \tau_{i}}{\partial X_{j}}\left(\Sigma, T, X_{j}\right)\right) \dot{X}_{j}=\frac{\partial \tau_{i}}{\partial \Sigma_{k l}}\left(\Sigma, T, X_{j}\right) \dot{\Sigma}_{k l}+\frac{\partial \tau_{i}}{\partial T}\left(\Sigma, T, X_{j}\right) \dot{T}
$$

whose resolution gives the evolution of internal variables as function of the thermomechanical load and therefore the inelastic response as well as the overall behavior of the single crystal using equation (10). 


\section{APPLICATION}

As a first application, the theoretical model developed in this work is applied in a simple case corresponding to a macroscopic shear in the $(1,3)$ plan of the external reference system. Only one variant is assumed to appear parallel to the shear stress and therefore the Magee effect is introduced. Furthermore, the plastic flow of austenitic and martensitic phases is described by a simple slip and the transformation strain is assumed purely deviatoric. Figure 1 shows the results for a shear test at constant temperature. The stressstrain curve $(\Sigma, E)$ OABC consists of four stages: elastic behavior of austenite (OA), plastic flow without transformation $(\mathrm{AB})$, plastic flow inside the austenite and martensitic phase transformation (BC), after $\mathrm{C}$, plasticity occurs in both phases. The dotted curve $(f, E)$ represents the evolution of the volume fraction of martensite. Figure 2 shows the evolution of $E$ tp as function of the.volume fraction $f$ during cooling at various applied stresses $\Sigma$. The TRIP effect is clearly reproduced since $E$ tp increases with $\Sigma$ at a fixed $f$ where the transformation strain ( $f \varepsilon t r$ ) is given despite the fact that $\Sigma$ is less than the austenitic yield stress. The obtained results agree qualitatively with the experimental ones performed by Gautier et al. [1] but not quantitatively since in the considered simple case the Magee effect is complete (all martensitic domains are oriented in the direction of the applied stress).

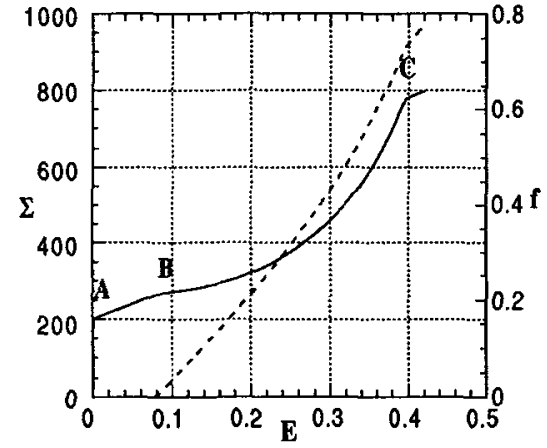

Fig. 1. Stress-strain curve of a Fe-Ni TRIP steel and the evolving volume fraction of martensitic phase

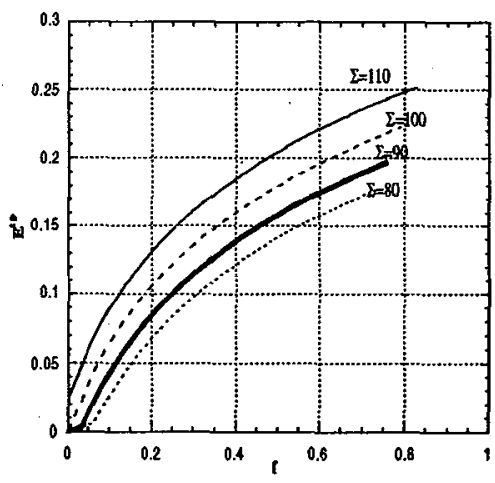

Fig. 2. Macroscopic inelastic strain in dependence of martensitic volume fraction and the level of external applied stress

\section{CONCLUSION}

Within a thermodynamic and micromechanic framework, a constitutive equation for an austenitic single crystal undergoing transformation induced plasticity has been derived. At a given stage of transformation and plastic flow inside both phases, the condition for the apparition of a martensitic domain inside the austenite with given transformation strain and aspect ratio is deduced. This transformation mechanism differs deeply from other conditions usually assumed in the literature. To check the model an application on a single variant and slip systems during a thermomechanical shear test shows results which are in good agreement with experiments. Non deviatoric effects, second order stress fluctuations (from austenitic grain to grain) and multivariant interactions which are present in the theoretical model will improve the numerical results.

\section{References}

[1] Gautier E. and Simon A., In Phase Transformation London 87 (1988) pp. 285-287.

[2] Magee C.L., Ph. D. Thesis Carnegie Institute of Technology Pittsburgh PA. (1966).

[3] Greenwood G.W. and Johnson R.H., Proc. Roy. Soc. A 283 (1967) 403.

[4] Leblond J.B., Devaux J. and Devaux J.C., Int. J. Plasticity 5 (1989) pp. 551-572.

[5] Fischer F.D. and Schlögl S.M., Mech. Mater. 21 (1995) pp. 1-23.

[6] Olson G.B. and Cohen M., Metall. Trans. A 6A (1975) 791.

[7] Stringfellow R.G., Parks, D.M. and Olson G.B., Acta Metall. Mater. 40 (1992) pp. 1703-1716.

[8] Bhattacharyya A. and Weng G.J., J. Mech. Phys. Solids 42 (1994) pp. 1699-1724.

[9] Wechsler M.S., Lieberman D.S. and Read T.A., AIME Trans. J. Metals 197 (1953) 1503.

[10] HILL R. ,J. Mech. Phys. Solids 31 (1983) 347.

[11] Sabar H., Buisson M. and Berveiller M., Int. J. Plast. 7 (1991) 759. 